\title{
Activation of Ciona sperm motility: phosphorylation of dynein polypeptides and effects of a tyrosine kinase inhibitor
}

\author{
CHINMOY S. DEY and CHARLES J. BROKAW* \\ Division of Biology 156-29, California Institute of Technology, Pasadena, California 91125, USA \\ * Author for correspondence
}

\section{Summary}

A high molecular mass dynein ATPase polypeptide and a $18-20 \mathrm{kDa}$ dynein light chain of Ciona sperm flagella are phosphorylated during in vivo activation of motility or in vitro activation of motility by incubation with cyclic AMP. A similar level of phosphorylation of these proteins is obtained by incubation of washed, demembranated spermatozoa with catalytic subunit of cyclic AMP-dependent protein kinase, under conditions where there is no activation of motility until a supernatant component is added. Therefore, phosphorylation of these dynein polypeptides is not sufficient for activation of motility. Activation of motility in vitro by incubation with cyclic AMP can be completely inhibited by a random copolymer of glutamate and tyrosine that inhibits tyrosine kinase activity. Under these conditions, much of the protein phosphorylation associated with activation of motility is also inhibited. These new results suggest that regulation of motility of these spermatozoa may involve a multicomponent kinase cascade rather than a simple phosphorylation of a protein 'switch' by the cyclic AMP-dependent kinase. A $53 \mathrm{kDa}$ axonemal phosphoprotein band, identified as band M1, shows the strongest correlation with activation of motility in these experiments.

Key words: cyclic AMP-dependent protein kinase, dynein, flagella, motility activation, spermatozoa, tyrosine kinase, protein phosphorylation.

\section{Introduction}

Cyclic AMP-dependent protein phosphorylation is necessary for activation of motility of spermatozoa of the tunicate, Ciona intestinalis (Brokaw, 1987a,b; Opresko and Brokaw, 1983), and has also been implicated in activation of motility of other spermatozoa, such as trout spermatozoa (Morisawa and Okuno, 1982; Tash, 1989). With Triton-demembranated Ciona spermatozoa, incubation with cyclic AMP and ATP converts the flagellar axoneme from a completely non-motile state to a state of full motility. During this incubation, many sperm flagellar proteins are phosphorylated, including a high molecular mass polypeptide that was suspected to be a dynein ATPase polypeptide, but no protein phosphorylations essential for motility have been positively identified (Opresko and Brokaw, 1983). In these investigations, H8, an inhibitor of cyclic AMP-dependent protein kinase (Hidaka et $a l$. 1984) that is readily membrane permeant, has been used with good effect to provide evidence that phosphorylation is required for activation in vivo as well as in vitro, in both Ciona and mammalian spermatozoa (Tash et al. 1986).

With trout spermatozoa, a $15 \mathrm{kDa}$ phosphoprotein was identified as a candidate for a regulator of motility activation (Morisawa and Hayashi, 1985). Subsequent work demonstrated that this $15 \mathrm{kDa}$ protein was phosphorylated on tyrosine residues, and that its cyclic AMPdependent phosphorylation in vitro was inhibited by a random copolymer of tyrosine and glutamate (poly-GluTyr [4:1], or PGT) that can serve as a competitive Journal of Cell Science 100, 815-824 (1991)

Printed in Great Britain (C) The Company of Biologists Limited 1991 substrate or competitive inhibitor of tyrosine phosphorylation (Hayashi et al. 1987).

We have followed up this work using improved methods for preparing, labelling and analyzing phosphoproteins of Ciona spermatozoa. In particular, we have examined the possibility that phosphorylations of dynein polypeptides are involved in activation of motility by examining high salt extracts that appear to remove outer dynein arms from Ciona spermatozoa. We have also examined the effects of PGT on protein phosphorylation and on in vitro activation of sperm motility in Ciona spermatozoa and obtained evidence that suggests the involvement of a tyrosine kinase in activation of motility of these spermatozoa.

Some of this work was presented previously in an abstract (Dey and Brokaw, 1989).

\section{Materials and methods}

\section{Sperm preparations}

Spermatozoa were collected by dissecting Ciona sperm ducts as described previously (Brokaw, 1982). The samples (up to $100 \mu$ of dry sperm) were diluted with $0.5 \mathrm{ml}$ of a wash solution that does not activate motility, containing $0.5 \mathrm{M} \mathrm{NaCl}, 50 \mathrm{mM} \mathrm{KCl}, 1 \mathrm{~mm}$ $\mathrm{CaCl}_{2}$ and $5 \mathrm{mM}$ Hepes buffer ( $\mathrm{pH} 7.6$ ), and then centrifuged at 2600 revs $\min ^{-1}(1000 \mathrm{~g})$ at $4{ }^{\circ} \mathrm{C}$. After centrifugation for 5 min the supernatant was removed carefully and the soft washed sperm pellet was stirred and kept on ice until used for experiments.

Isolation of flagella

A $100 \mu \mathrm{l}$ sample of washed spermatozoa was transferred to a 
small glass culture tube, broken by using a high-speed vortex mixer for $20 \mathrm{~s}$ and then diluted $1: 7$ with $0.5 \mathrm{M} \mathrm{NaCl}$ containing $50 \mathrm{~mm} \mathrm{KCl}, 2 \mathrm{~mm}$ Tris-HCl $(\mathrm{pH} 8.2)$ and protease inhibitors (5 mM $\beta$-mercaptoethanol, $0.5 \mathrm{~mm}$ phenylmethylsulfonyl fluoride, and $0.2 \mathrm{~mm} \mathrm{~L} 1$-tosylamide 2-phenylethyl chloromethyl ketone). This diluted sample was layered on top of $4 \mathrm{ml}$ of a $25 \%$ sucrose solution containing $0.5 \mathrm{M} \mathrm{NaCl}, 50 \mathrm{mM} \mathrm{KCl}, 10 \mathrm{mM} \mathrm{MgSO}_{4}, 10 \mathrm{~mm}$ Tris- $\mathrm{HCl}(\mathrm{pH} 7.6)$ and protease inhibitors, and centrifuged at

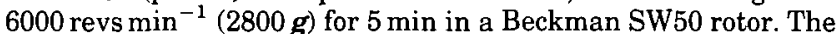
white layer of sperm tails on the top of the sucrose solution was removed and centrifuged at 10000 revs $\min ^{-1}(6600 \mathrm{~g})$ for $8 \mathrm{~min}$ in a Beckman no. 40 ultracentrifuge rotor. The flagellar pellet was resuspended in 1 to $2 \mathrm{ml}$ of wash solution, centrifuged again at $6600 \mathrm{~g}$ for $6 \mathrm{~min}$, and the resulting flagellar pellet was used for experiments. This procedure was carried out at 0 to $4^{\circ} \mathrm{C}$.

\section{Demembranation and reactivation of sperm motility}

These procedures were carried out at $18^{\circ} \mathrm{C}$. Washed spermatozoa were diluted 1:50 with demembranation solution containing $250 \mathrm{~mm} \mathrm{KCl}, 10 \mathrm{~mm}$ Tris-HCl, $10 \mathrm{~mm}$ Hepes, $1 \mathrm{~mm}$ dithiothreitol (DTT), $1 \mathrm{mM} \mathrm{MgSO}$, $0.4 \mathrm{~mm}$ EGTA and $0.04 \%$ Triton X-100 at $\mathrm{pH}$ 7.6. After $30 \mathrm{~s}$ the suspension was diluted with 1.5 volumes of a solution for activation of motility, containing the same concentrations of buffer, DTT and $\mathrm{MgSO}_{4}$, in addition to $0.2 \mathrm{mM}$ EGTA, $100 \mu \mathrm{M}$ ATP and $10 \mu \mathrm{M}$ cyclic AMP. After incubation for $2 \mathrm{~min}$, motility was reactivated by diluting $1: 500$ in reactivation solution containing $250 \mathrm{~mm}$ potassium acetate, $10 \mathrm{mM}$ Tris $-\mathrm{HCl}, 10 \mathrm{~mm}$ Hepes, $1 \mathrm{~mm}$ DTT, $1.5 \mathrm{~mm} \mathrm{MgSO}_{4}, 1.24 \mathrm{~mm}$ ATP, $0.5 \mathrm{~mm}$ EGTA and $0.5 \%$ polyethyleneglycol at $\mathrm{pH}$ 7.6. This procedure, involving in vitro activation, is similar to previously published work from this laboratory (Brokaw, 1985), except that recent work has determined that a lower $\mathrm{pH}$ is optimal for reactivation of Ciona spermatozoa (Brokaw, 1992). For some experiments, the Triton-supernatant was removed from the demembranated spermatozoa by centrifuging the suspension for $10 \mathrm{~min}$ at 10000 revs $\mathrm{min}^{-1}(8200 \mathrm{~g})$ in an Eppendorf microcentrifuge and then resuspending the sperm pellet in demembranation solution without Triton X-100.

Activation of motility in vivo was performed by diluting washed spermatozoa $1: 10$ with a solution consisting of $90 \%$ of a solution of $0.55 \mathrm{M} \mathrm{NaCl}$ containing $80 \mathrm{~mm}$ histidine, $1 \mathrm{mM} \mathrm{CaCl}_{2}$, and $5 \mathrm{~mm}$ theophylline, and $10 \%$ filtered sea water, at $\mathrm{pH} 8.4$ (Brokaw, $1987 a, b)$. After $30 \mathrm{~s}$ of incubation, this mixture was diluted 1:10 with demembranation solution, as described in the preceding paragraph. Activation can be assayed by diluting this mixture directly into reactivation solution.

\section{Preparation of high concentration Triton extract (HTE)}

Washed spermatozoa were diluted 1:10 with a demembranation solution with the Triton concentration increased to $0.1 \%$ and the EGTA concentration increased to $1.4 \mathrm{~mm}$. After demembranating for $30 \mathrm{~s}$ at $18^{\circ} \mathrm{C}$, the mixture was centrifuged in a Beckman no. 40 ultracentrifuge rotor at $20000 \mathrm{revs} \mathrm{min}^{-1}(26000 \mathrm{~g})$ for $5 \mathrm{~min}$ at $4^{\circ} \mathrm{C}$. The supernatant $(\mathrm{HTE})$ was stored at $0^{\circ} \mathrm{C}$

\section{${ }^{32} P$ labelling of sperm proteins}

Proteins phosphorylated during incubation with cyclic AMP and ATP under our standard conditions for in vitro activation were identified by labelling with ${ }^{32} \mathrm{P}$, followed by polyacrylamide gel electrophoresis (SDS-PAGE) and autoradiography. Each gel sample was prepared separately, using $2 \mu \mathrm{l}$ of washed spermatozoa or flagella, with $10 \mu \mathrm{Ci}$ of $\left.{ }^{32} \mathrm{P}\right] \mathrm{ATP}$ added to the activation solution in addition to the normal $100 \mu \mathrm{M}$ ATP. At the end of the 2 min incubation period at $18^{\circ} \mathrm{C}$, sodium pyrophosphate and unlabelled ATP, to concentrations of $15 \mathrm{mM}$ and $2 \mathrm{mM}$, respectively, were added to stop the reaction, and the samples were placed on ice. Each sample was centrifuged, and the pellets were prepared for SDS-PAGE. Supernatant proteins were precipitated with $10 \%$ trichloroacetic acid, and the pellets were prepared for SDS-PAGE. Proteins phosphorylated after in vivo activation were examined in a similar manner, by preparing demembranated, in vivo activated spermatozoa as described above, and then mixing with in vitro activation solution containing labelled ATP.

In all of these experiments with intact spermatozoa, parallel samples prepared with the same washed sperm pellet and solutions, but without the addition of $\left[{ }^{32} \mathrm{P}\right] \mathrm{ATP}$, were reactivated and examined, to confirm that the activation of motility was normal. This control is not possible with preparations of isolated flagella, which cannot be reactivated when prepared by shearing off the sperm heads.

\section{Preparation and sucrose density gradient analysis of high salt extract}

Sperm samples were demembranated, activated in vitro with or without $\left[{ }^{32} \mathrm{P}\right] \mathrm{ATP}$, arrested, and centrifuged as described above. The pellet was then extracted with $0.6 \mathrm{M} \mathrm{NaCl}$ in a mixture of $100 \mu \mathrm{l}$ of demembranation solution without Triton and $150 \mu \mathrm{l}$ of activation solution without cyclic AMP or ATP, containing $15 \mathrm{~mm}$ sodium pyrophosphate. After $30 \mathrm{~s}$ of extraction at $0^{\circ} \mathrm{C}$, the samples were centrifuged. The pellets were prepared for SDSPAGE. The supernatants ( $250 \mu \mathrm{l}$ each) were concentrated to $60 \mu \mathrm{l}$ by ultrafiltration with a Millipore centrifugal concentrator (100000 MW) and then prepared for SDS-PAGE.

A larger-scale preparation of $\mathrm{NaCl}$ extract from axonemes was used to prepare samples for analysis by sucrose density gradient centrifugation. Using a scaled-up procedure, flagella were isolated from $500 \mu \mathrm{l}$ of washed spermatozoa. These flagella were demembranated, activated (in some experiments in the presence of $200 \mu \mathrm{Ci}$ of $\left[{ }^{32} \mathrm{P}\right] \mathrm{ATP}$ ), extracted with $0.6 \mathrm{M} \mathrm{NaCl}$, and centrifuged as in the preceding paragraph. Protease inhibitors were added to the $18.75 \mathrm{ml}$ of supernatant, which was then concentrated to $250 \mu \mathrm{l}$ by ultrafiltration; $200 \mu \mathrm{l}$ was placed on top of a $4 \mathrm{ml}$ linear sucrose density gradient (5\% to $30 \%$ ) made up in a solution containing $100 \mathrm{~mm} \mathrm{KCl}, 10 \mathrm{~mm}$ Tris- $\mathrm{HCl}, 10 \mathrm{~mm}$ Hepes, $1 \mathrm{~mm}$ DTT, $1 \mathrm{~mm} \mathrm{MgSO}$, $0.275 \mathrm{~mm}$ EGTA and protease inhibitors. Centrifugation was for $15 \mathrm{~h}$ at 25000 revs $\mathrm{min}^{-1}(51000 \mathrm{~g})$ in a Beckman SW 50 rotor at $4^{\circ} \mathrm{C}$. Fractions of $0.2 \mathrm{ml}$ were collected from the top of each tube, using an ISCO density gradient fractionator and tube piercer. Samples $(20 \mu \mathrm{l})$ of each fraction were prepared for SDS-PAGE. During collection, protein concentration was monitored at $280 \mathrm{~nm}$ with an ISCO UA-5 absorbance monitor. The gradients were calibrated with identical runs using collagenase (5.7 S) and thyroglobulin (19 S) as standards.

\section{ATPase assays}

Extraction of ATPase activity by $0.6 \mathrm{M} \mathrm{NaCl}$ was monitored using samples prepared in the same manner as described above, without ultrafiltration; $200 \mu \mathrm{l}$ of extract, $100 \mu \mathrm{l}$ of resuspended pellet, or $100 \mu$ of the uncentrifuged $0.6 \mathrm{M} \mathrm{NaCl}$-extracted spermatozoa was added to $10 \mathrm{ml}$ of assay solution containing $250 \mathrm{~mm}$ potassium acetate, $10 \mathrm{~mm}$ Tris $-\mathrm{HCl}, 10 \mathrm{~mm}$ Hepes, $1 \mathrm{~mm}$ DTT, $1.5 \mathrm{~mm}$ $\mathrm{MgSO}_{4}, 0.6 \mathrm{M} \mathrm{KCl}, 0.62 \mathrm{~mm}$ ATP at $\mathrm{pH} 7.6$. The reaction mixture was incubated at $18^{\circ} \mathrm{C}$. At times of 1,4 and $7 \mathrm{~min}, 1.5 \mathrm{ml}$ samples were removed and immediately mixed with the reagents for measuring inorganic phosphate by a malachite green method (Carter and Karl, 1982). Distribution of ATPase activity following sucrose density gradient centrifugation was monitored in a similar manner by assaying $150 \mu \mathrm{l}$ samples from each $200 \mu \mathrm{l}$ fraction and measuring the inorganic phosphate released after 4 min of incubation.

\section{Assays of protein kinase activity using exogenous substrates}

Assays of phosphorylation by the catalytic subunit of cyclic AMPdependent protein kinase were performed using exogenous substrates, histone, casein or kemptide, to examine the effects of inhibitors on this reaction. These assays used an assay solution with the same composition as the incubation mixtures used for activation and phosphorylation of spermatozoa, except that cyclic AMP was omitted. With the protein substrates, the reaction was arrested by addition of excess casein, $10 \mathrm{~mm}$ unlabelled ATP and $205 \mathrm{~mm}$ potassium phosphate. The proteins were then precipitated with trichloroacetic acid, collected on $0.45 \mu \mathrm{m}$ membrane filters (Type HA, Millipore Corp., Bedford MA), washed with 5\% trichloroacetic acid, dried and counted for ${ }^{32} \mathrm{P}$ radioactivity. With the peptide substrate, the reaction was arrested by addition of glacial acetic acid. Samples were removed and spotted on 
Whatman P81 filter discs, which were then dried, washed and counted for ${ }^{32} \mathrm{P}$ radioactivity (de la Houssaye and Masaracchia, 1983).

\section{Immunoblotting}

Transfer of proteins from the gel to the nitrocellulose paper was done according to King et al. (1985). A polyclonal antiphosphotyrosine was a gift from $\mathrm{Dr} \mathrm{W}$. Kinsey (Peaucellier et al. 1988).

\section{Protein estimation}

Protein concentrations were measured with the Coomassie blue dye binding method by using the BioRad reagent with BSA as routine standard.

\section{SDS-PAGE and autoradiography}

SDS-PAGE was done according to Laemmli (1970). Gels were stained with silver nitrate using the method of Morrissey (1981). Dried gels were exposed to Kodak XAR5 film at $-70^{\circ} \mathrm{C}$, with or without intensifier screens. Densitometry was performed by using a digitizing camera (model EC850, Eikonix Corp., Bedford, MA) interfaced with a Hewlett Packard model 320 microcomputer to record 256 grey levels, with a band length of 2048 pixels per $11 \mathrm{~cm}$ of autoradiogram and a band width of 16-128 pixels. After conversion of the grey scale values to optical density, the density distribution was modeled by trial and error with a set of 34 Gaussian peaks (Kitazoe et al. 1983). The linearity of the relationship between peak area and ${ }^{32} \mathrm{P}$ radioactivity was verified with an autoradiogram of a gel prepared with a series of known dilutions of a sperm extract containing phosphoproteins. Since most of the autoradiograms contained many overlapping peaks, it was not possible to establish local baselines accurately, and the results must be considered to be only approximate. The ratios of peak areas given in Table $I$ are the mean values from two (lines (a),(c),(d)) or three (line (b)) experiments; in the latter case, standard errors ranged from 0.06 to 0.09 , except for band D1 where the mean had a standard error of 0.24 .

\section{Reagents}

Protein kinase catalytic subunit from bovine brain (P2645), PGT (P0275), PGA (P1650), casein (C6780), histone (H5505), kemptide (K1127) and EGTA were obtained from Sigma Chemical Co., St Louis MO. H8 was from Seikagaku America Inc., St Petersburg FL. ATP was from Boehringer-Mannheim Biochemicals, Indianapolis, IN. ${ }^{32}$ P]ATP $\left(3 \times 10^{6} \mathrm{Ci} \mathrm{mol}^{-1}\right)$ was from Amersham Corp., Arlington Heights, IL.

Table 1. Results from densitometry of ${ }^{32} \mathrm{P}$ autoradiograms

\begin{tabular}{|c|c|c|c|c|c|c|c|c|}
\hline \multirow[b]{2}{*}{ Experiment } & \multicolumn{8}{|c|}{ Phosphoprotein band identifier } \\
\hline & A & D1 & $\mathrm{F}$ & $\mathrm{K}$ & M1 & M2 & $Q$ & $\mathrm{R}$ \\
\hline $\begin{array}{l}\text { (a) Standard incubation } \\
\text { protocol (Fig. 1); } \\
\text { PGT sample/ } \\
\text { PGA sample }\end{array}$ & 0.10 & 0.32 & 0.21 & 0.28 & 0.21 & 0.38 & 0.65 & 0.50 \\
\hline $\begin{array}{l}\text { (b) Washed spermatozoa } \\
\text { and exogenous kinase; } \\
\text { PGT sample/ } \\
\text { PGA sample }\end{array}$ & 0.35 & 0.75 & & 0.55 & & 0.52 & 058 & 0.51 \\
\hline $\begin{array}{l}\text { (c) In vivo activation/ } \\
\text { in vitro activation } \\
\text { (Fig. } 5 \text { ) }\end{array}$ & 0.12 & 0.17 & 0.19 & 0.18 & 0.13 & 0.19 & 0.40 & 0.23 \\
\hline $\begin{array}{l}\text { (d) Washed spermatozoa } \\
\text { preincubated for } 30 \mathrm{~s} \\
\text { with exogenous kinase/ } \\
\text { no preincubation } \\
\text { (Fig. 6) }\end{array}$ & 0.24 & 0.38 & 0.76 & 036 & 0.75 & 0.69 & 0.48 & 0.48 \\
\hline
\end{tabular}

\section{Results}

Phosphorylation of dynein polypeptides during in vitro activation of sperm motility

Results of incubation of whole demembranated spermatozoa with cyclic AMP and labelled ATP under standard conditions are illustrated by the lanes labelled S in Figs 1 and 5 . Under these conditions, sperm motility is fully activated, with almost $100 \%$ of the spermatozoa swimming with propagated bending waves, as described (Brokaw, 1987b). The autoradiogram in Fig. 1 indicates that at least 36 distinct polypeptides are labelled under these conditions. Our previous work (Opresko and Brokaw, 1983) and new results (not shown) have indicated that almost all of this phosphorylation, as well as activation of motility, is dependent upon the addition of cyclic AMP to the incubation mixture. Opresko and

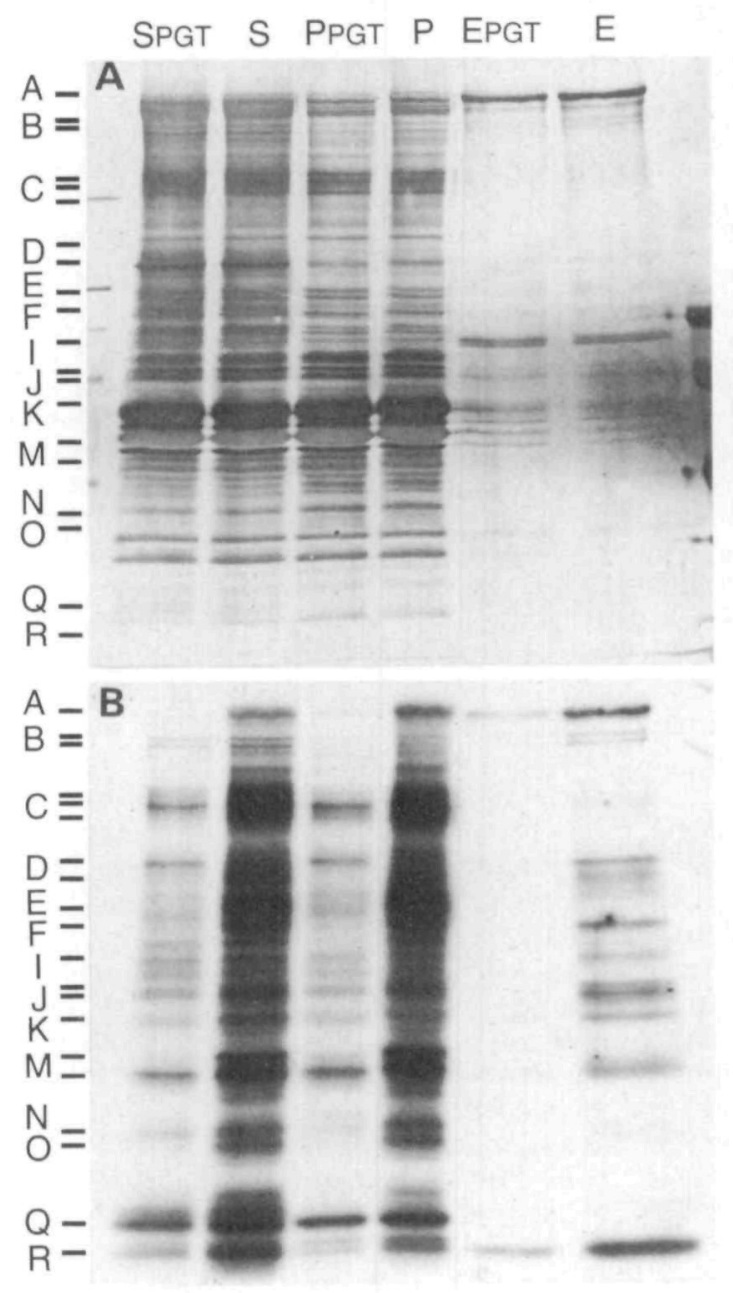

Fig. 1. Polyacrylamide gel electrophoresis (A) and ${ }^{32} \mathrm{P}$ autoradiogram (B) of samples from whole demembranated spermatozoa (S), pellets after $0.6 \mathrm{M} \mathrm{NaCl}$ extraction (P), and $0.6 \mathrm{M} \mathrm{NaCl}$ extracts (E). Lanes labelled PGT contained $20 \mu \mathrm{g} \mathrm{ml}^{-1}$ poly-Glu-Tyr during the incubation with cyclic AMP and ATP. The control lanes contained $20 \mu \mathrm{g} \mathrm{ml}^{-1}$ poly-Glu-Ala during the incubation with cyclic AMP and ATP.

Phosphoprotein bands are identified with letter codes, on the left, corresponding to the letter identifications assigned by Opresko and Brokaw (1983). Molecular mass standards are labelled in Fig. 5. 
Brokaw (1983) applied letter designations to 15 phosphoprotein bands seen in similar autoradiograms. We have used the same scheme, with the assignments shown in Fig. 1. Several of the bands designated previously (bands B, C, D, E, J, M) are now clearly represented by multiple bands; where necessary we identify these by adding numbers (e.g. D1, D2; D2 is the band D referred to by Opresko and Brokaw (1983)). In most cases, the correlation of the bands seen here with those in the earlier autoradiograms is straightforward. However, bands $\mathrm{J}$ and $\mathrm{K}$ are problematic. The appearance and behavior of the bands labelled $\mathrm{J}$ and $\mathrm{K}$ in Fig. 1 resembles the correspondingly labelled bands of Opresko and Brokaw (1983), but in our gels these two bands are found at lower $M_{\mathrm{r}}$ values, with band $\mathrm{J}$ rather than $\mathrm{K}$ at approximately $65 \times 10^{3}$ and band $\mathrm{K}$ close to the location of the uppermost heavily stained band seen in the tubulin region of the gels.

Fig. 1 shows the results of extracting the demembranated and activated spermatozoa with $0.6 \mathrm{M} \mathrm{NaCl}$, followed by centrifugation to obtain pellet $(\mathrm{P})$ and high salt extract (E) fractions. Concentration of the extract by ultrafiltration could have reduced the relative concentration of low molecular mass components in the extract while concentrating large components such as dynein. None of the major gel bands appears to be missing from both $\mathrm{P}$ and $\mathrm{E}$ fractions, but one indistinct phosphoprotein band visible in lane $S$ just above the band labelled $Q$ appears to be extracted from $P$ without appearing in $E$. The predominant component in these extracts is represented by a high molecular mass band visible in the gels and by phosphoprotein band $\mathrm{A}$ in the autoradiograms, which is expected to represent heavy chains of dynein ATPase. Neither these gels, nor 3-6\% gels (not shown) with similar protein loadings resolve this high molecular mass band into $\alpha$ and $\beta$ bands as typically seen for outer arm dynein from sperm flagella (Bell et al. 1982). In similar experiments, measurements of ATPase activity of the extract, pellet, and uncentrifuged high salt mixture were compared, giving values of $9.8 \pm 0.5,8.0 \pm 0.3$ and $25 \pm 2$ nanomol $P_{\mathrm{i}} \min ^{-1} \mu \mathrm{l}^{-1}$ of spermatozoa, respectively (mean and standard error for 3 experiments). Approximately $20 \%$ of the protein was found in the extract, and in all three fractions, approximately $50 \%$ of the ATPase activity was inhibited by $100 \mu \mathrm{M}$ vanadate. In the high molecular mass region of lane $\mathrm{P}$ of the autoradiogram, the labelling appears to extend above the position of the prominent high molecular mass polypeptide band, suggesting that there is phosphorylation of a residual high molecular mass polypeptide that is not the same as the phosphoprotein that is extracted by $0.6 \mathrm{M} \mathrm{NaCl}$. This pattern was seen consistently.

Another prominent component of the extract, seen on the gel, is a pair of bands at approximately $85 \mathrm{kDa}$. These bands are at approximately the location of a weak phosphoprotein band, band I. The other very prominent phosphoprotein in the extracts is band $R$, which is represented by only a relatively weak phosphoprotein band in the pellet fraction. No gel band corresponding to phosphoprotein band $\mathrm{R}$ can be detected.

The samples shown in Fig. 1 were prepared from whole spermatozoa, rather than flagella, in order to match as closely as possible the conditions where activation of motility can be confirmed. Gels and autoradiograms obtained using isolated flagella, rather than whole spermatozoa, gave rather similar results (Opresko and Brokaw, 1983) with bands $\mathrm{A}, \mathrm{C}(\mathrm{C} 2$ ?), D2, K, M1, Q and possibly $R$ clearly identified as axonemal components.

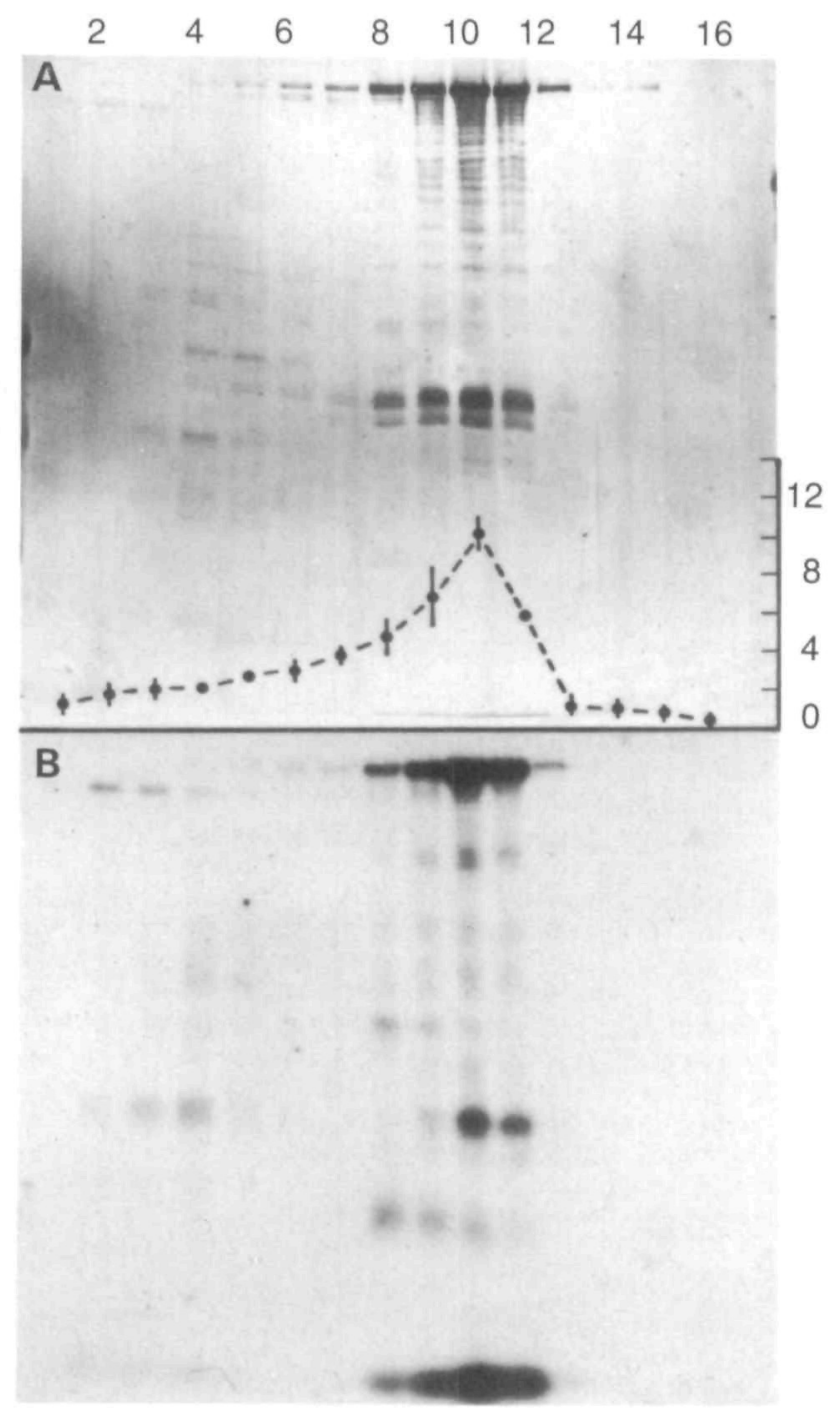

Fig. 2. Polyacrylamide gel electrophoresis (A) and ${ }^{32} \mathrm{P}$ autoradiogram (B) of fractions from sucrose density gradient centrifugation of $0.6 \mathrm{M} \mathrm{NaCl}$ extract of axonemes from Ciona sperm flagella following incubation with cyclic AMP and ATP. Fractions are numbered from the top of the gradient. The curve in A shows ATPase measurements (mean and S.E. for 3 samples) from sucrose density gradient centrifugations under identical conditions, but with unlabeled ATP. Units are $10^{-8}$ mol $\min ^{-1}$ per $200 \mu \mathrm{l}$ fraction.

Isolated flagella were used to prepare extracts for analysis by sucrose density centrifugation, and a typical result is shown in Fig. 2. The distribution of protein in the gradient was monitored by UV absorbtion during fractionation. A single prominent protein peak was observed that was consistently centered between fractions 9 and 10, slightly ahead of the position of the thyroglobulin peak (19 S). ATPase activity peaked in fraction 10 , as shown by the data curve overlaying the gel photograph in Fig. 2A. The predominant high molecular mass polypeptide in the extracts also appears in fractions 8-11, with a peak in fraction 10 , and its distribution is identical to that of phosphoprotein band A. This high molecular mass band appears to be resolved into $\alpha$ and $\beta$ bands when its concentration is low, as in fractions 7 and 14 , but the resolution of these autoradiograms is not sufficient to 
determine whether the ${ }^{32} \mathrm{P}$ labelling coincides with one or both of these bands.

Several other components appear to comigrate in the sucrose density gradient with the high molecular mass peptide corresponding to phosphoprotein band A. These include two or more polypeptides visible in the gel in the $70-85 \mathrm{kDa}$ region, which were also seen in Fig. 1, lane E. The gels also show many minor bands below the main high molecular mass component that could result from proteolysis of high molecular mass bands. In the autoradiogram of Fig. 2B, the phosphoprotein band $\mathrm{R}$ clearly comigrates with the high molecular mass peptide corresponding to phosphoprotein band A. Another phosphoprotein in the middle of the gel, which may correspond to band J, appears to consistently migrate slightly ahead of these two components, suggesting heterogeneity of dynein components that should be investigated further.

Together, these results suggest that phosphoprotein bands $A$ and $R$ are components of a dynein ATPase particle, with band $A$ representing phosphorylation of one or both of the high molecular mass ATPase polypeptides, and band $R$ representing equally strong phosphorylation of a dynein light chain with a molecular mass in the $18-20 \mathrm{kDa}$ range. Densitometry of autoradiograms such as Fig. 1, lane S, indicate that approximately $3 \%$ of the ${ }^{32} \mathrm{P}$ incorporation is in band $\mathrm{A}$. In the earlier ${ }^{32} \mathrm{P}$ labelling experiments by Opresko and Brokaw (1983), the total phosphorylation under conditions similar to those used here was approximately 30 to $60 \mathrm{pmol} / 10^{9}$ spermatozoa. Each sperm flagellum contains approximately 32000 dynein arms, based on a flagellar length of $48 \mu \mathrm{m}$ and 7 or 8 dynein arms per $96 \mathrm{~nm}$ of 8 or 9 outer doublet microtubules. These values indicate that ${ }^{32} \mathrm{P}$ incorporation into dynein heavy chains corresponding to approximately 0.03 to $0.06 \mathrm{~mol}$ of phosphate per mol of dynein occurs during in vitro activation of Ciona sperm motility. ${ }^{32} \mathrm{P}$ incorporation into band $R$ is somewhat higher, approximately $7 \%$ of the total, but no information about the stoichiometry of this dynein light chain is available.

$P G T$ inhibits activation of Ciona sperm motility and phosphorylation of many sperm proteins

Observations of reactivated sperm motility were made using two random copolymers of amino acids. PGT, a polymer of glutamic acid and tyrosine, can act either as a competitive substrate for or an inhibitor of tyrosine kinase (Braun et al. 1984). PGA, a polymer of glutamic acid and alanine, was used as a control. Fig. 3 shows that addition of PGT to the cyclic AMP-ATP activation solution at a concentration as low as $20 \mu \mathrm{g} \mathrm{ml}^{-1}$ completely inhibited activation of Ciona sperm motility, whereas PGA at concentrations up to $100 \mu \mathrm{g} \mathrm{ml}^{-1}$ had no effect.

Phosphorylation of proteins during cyclic AMP-dependent activation of sperm motility in the presence of $20 \mu \mathrm{g} \mathrm{ml}^{-1}$ of either PGA or PGT was examined by autoradiography (Fig. 1). There was no significant-difference in the pattern of labelling in the presence or absence of PGA (compare lanes $\mathrm{S}$ in Fig. 1 and Fig. 5). PGT strongly inhibited the phosphorylation of band $A$ and many of the other prominent phosphoproteins, but a few bands, such as C2, D1, M2 and $Q$, continue to show substantial phosphorylation in the presence of PGT. Table 1, line (a) summarizes quantitation of some of these phosphoprotein bands.

To interpret these results, it was necessary to consider whether PGT is a general inhibitor of cyclic AMPdependent protein kinase. Assays were carried out with

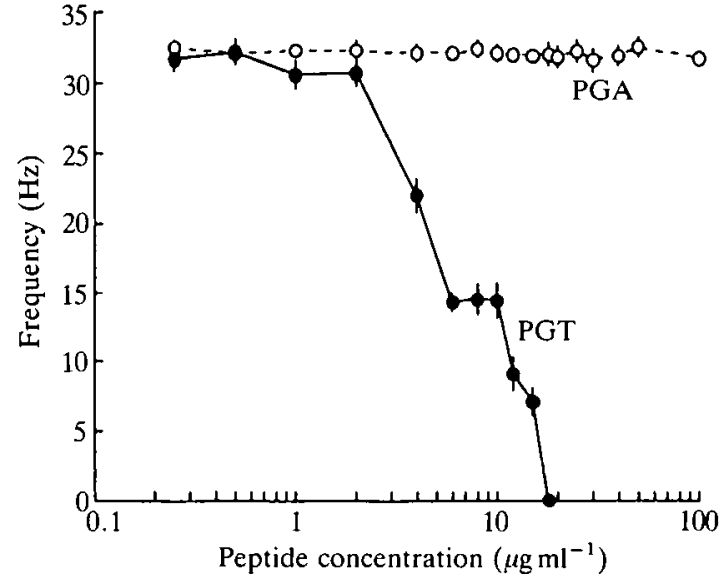

Fig. 3. Inhibition of the activation of motility by a tyrosine kinase inhibitor, PGT (filled circles) is compared with normal activation obtained with a control peptide, PGA (open circles). Flagellar beat frequency was measured after diluting a sample of the activation mixture, containing peptide at the concentration shown, into a 500-fold greater volume of reactivation solution. Each point shows the mean and standard deviation for 10 spermatozoa. At $18 \mu \mathrm{g} \mathrm{ml}^{-1}$ PGT or higher, only sporadic movement, with no regular oscillation of the flagellum, is seen.

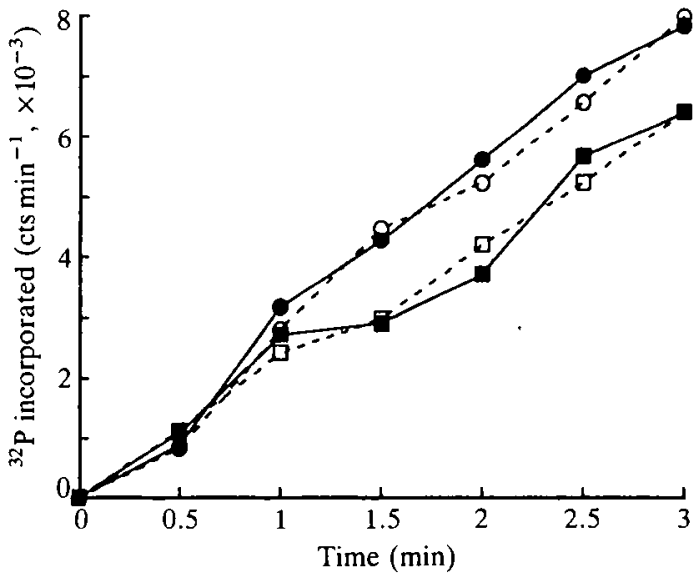

Fig. 4. Effects of PGT (filled symbols) and PGA (open symbols) on ${ }^{32} \mathrm{P}$ incorporation into proteins using catalytic subunit of cyclic AMP-dependent protein kinase. $(0,0)$ Obtained with histone as substrate; $(\square, \square)$ with casein as substrate. Each point is the mean of two samples.

the catalytic subunit of cyclic AMP kinase using the exogenous protein substrates histone and casein. There was no inhibition of phosphorylation of these proteins in the presence of PGT at a concentration as high as $0.5 \mathrm{mg} \mathrm{ml}^{-1}$ (Fig. 4). Assays were also carried out using a synthetic peptide, kemptide, that is considered to be a very specific substrate of cyclic AMP-dependent protein kinase. These assays were carried out both with the catalytic subunit of AMP kinase and with demembranated sperm suspensions. In both cases, strong inhibition of substrate phosphorylation was obtained with $\mathrm{H} 8$, an inhibitor of cyclic AMP-dependent protein kinase (Hidaka et al. 1984), at a concentration of $1 \mathrm{mM}$, and no inhibition was obtained with PGT or PGA, at concentrations up to $0.5 \mathrm{mg} \mathrm{ml}^{-1}$ (data not shown). 
An attempt was made to detect flagellar protein(s) phosphorylated at tyrosine residues by immunoblotting with anti-phosphotyrosine antibodies. Immunoblots of samples prepared from demembranated and activated sperm pellets or supernatants, or similar pellet preparations from isolated flagella, were examined using a polyclonal anti-phosphotyrosine antibody. Some labelled bands were found, and some of these corresponded to bands seen in the $\left[{ }^{32} \mathrm{P}\right]$ ATP-labelling experiments (data not shown). However, the intensities of these bands did not differ between samples incubated with or without cyclic AMP, or with or without PGT. Since these bands behaved simply as constitutive phosphotyrosine-containing proteins, this analysis failed either to confirm or to identify changes in tyrosine phosphorylation occuring during activation of Ciona sperm motility.

\section{Phosphorylation after in vivo activation of motility}

Although the phosphorylation protocols followed in the experiments described above examine phosphorylation that occurs at the same time as activation of sperm motility, the conditions for this in vitro activation and phosphorylation may not duplicate the conditions obtained in vivo during normal activation of motility, and therefore could give a misleading picture of the phosphorylations that are important for activation of motility. To circumvent this problem, Opresko and Brokaw (1983) carried out 'reverse phosphorylation' experiments in which spermatozoa were activated in vivo before demembranation and incubation with cyclic AMP and ${ }^{32}$ P]ATP This protocol identified two phosphoproteins, bands A and $R$, that showed the greatest reduction in ${ }^{32} \mathrm{P}$ labelling, indicating extensive phosphorylation during the prior in vivo activation.

We have repeated these experiments, using an improved procedure for in vivo activation that requires only a $15 \mathrm{~s}$ incubation of the spermatozoa in activation solution before they are demembranated, in contrast to the more lengthy procedure of Opresko and Brokaw (1983) that required more extensive dilution followed by centrifugation to concentrate the spermatozoa before demembranation. A typical result is shown in Fig. 5; results of densitometry are given in Table 1, line (c). In these experiments, lower values in Table 1 indicate stronger correlation with motility. These results confirm the earlier conclusion that many sperm proteins become phosphorylated during in vivo activation and therefore show much less labelling during subsequent incubation with cyclic AMP and $\left[{ }^{32} \mathrm{P}\right]$ ATP. These phosphoproteins include bands A, M1 and $\mathrm{R}$. Phosphoprotein band $\mathrm{M} 1$, in particular, which was not separately identified in the previous study (Opresko and Brokaw, 1983), now appears to be one of the bands that is most thoroughly phosphorylated during in vivo activation. The new results identify band $Q$ as a band that can still be substantially phosphorylated following in vivo activation, suggesting that phosphorylation of this protein is less likely to be important for activation of motility.

\section{Phosphorylation after prephosphorylation with exogenous} kinase

In the previous study (Opresko and Brokaw, 1983) it was shown that removal of the Triton supernatant from demembranated spermatozoa eliminated their ability to activate motility, even in the presence of exogenous kinase - the catalytic subunit of the cyclic AMP-dependent protein kinase. Activation of motility could be restored by addition of the supernatant fraction, containing Triton

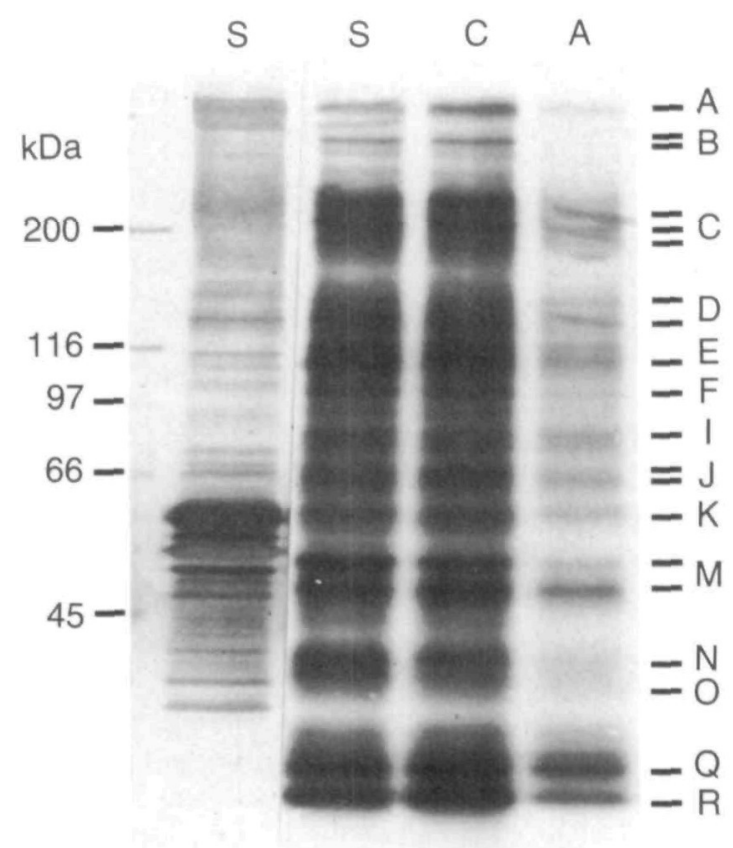

Fig. 5. Effect of in vivo activation of motility before demembranation and incubation with cyclic AMP and labelled ATP. Lane S shows a sample (gel and autoradiogram) prepared under standard in vitro activation conditions (the same as lane $\mathrm{S}$ of Fig. 1, except for the absence of PGA during incubation). Lane A shows an autoradiogram obtained from spermatozoa that were activated in vivo by dilution with a $\mathrm{pH} 8.4$ activation solution containing histidine and theophylline before

demembranation; the sample was then incubated with cyclic AMP and labelled ATP as in the standard in vitro activation procedure. The control lane (C) shows an autoradiogram obtained from spermatozoa prepared by a similar protocol, except that they were diluted with wash solution, which does not activate motility, instead of the $\mathrm{pH} 8.4$ activation solution. Phosphoprotein bands are identified by letters, and values for the molecular mass standards are shown (in $\mathrm{kDa}$ ) on the left.

$\mathrm{X}-100$ and extracted components, that was removed by centrifugation. This result was exploited to show that incubation with cyclic AMP or exogenous kinase and ${ }^{32} \mathrm{P}$ in the absence of the supernatant fraction resulted in labelling of a subset of the proteins labelled under standard conditions. In particular, the labelling of bands $\mathrm{E}, \mathrm{F}, \mathrm{H}, \mathrm{M}, \mathrm{N}$ and $\mathrm{O}$ was clearly reduced during incubation in the absence of the supernatant fraction, suggesting that these bands might be particularly important for activation of motility. Similar experiments under our new conditions confirm these observations, and also show that the phosphorylations that occur when washed spermatozoa are incubated with exogenous kinase are also sensitive to PGT (Table 1, line (b)).

We have now carried out complementary experiments designed to identify proteins that become phosphorylated only when motility is activated. Demembranated spermatozoa were centrifuged and resuspended in a medium similar to the standard activation medium, except for the absence of Triton X-100 and cyclic AMP, and the addition of protein kinase catalytic subunit at 2 units $\mu l^{-1}$. Following incubation for periods up to $5 \mathrm{~min}$, no activation of motility was observed when these spermatozoa were diluted directly into reactivation solution. However, when a second 2 min incubation period was added, following the addition of $10 \mu \mathrm{l}$ of a high Triton extract of spermatozoa 


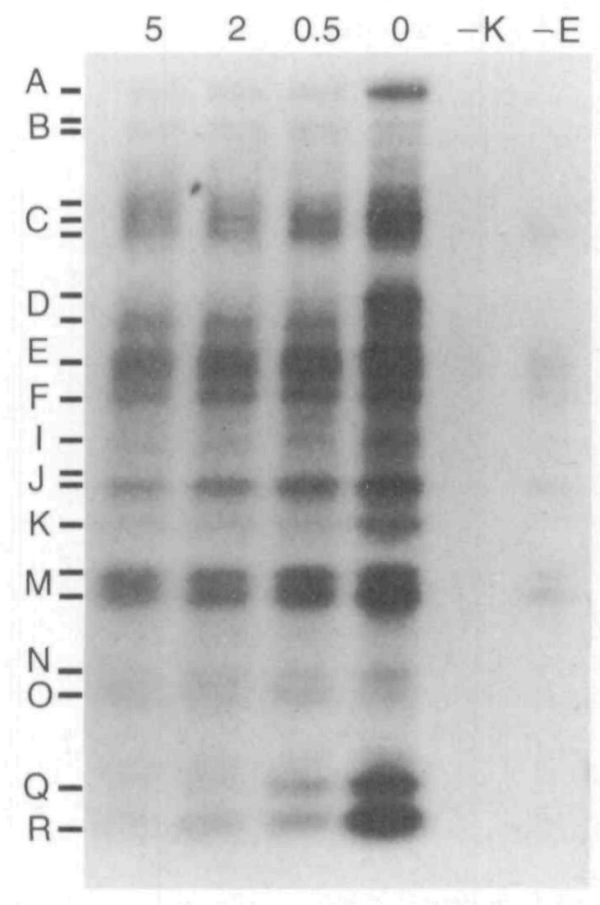

Fig. 6. Effect of preincubation of washed demembranated spermatozoa with protein kinase catalytic subunit on phosphorylation occuring during in vitro activation of motility After demembranation, the spermatozoa were centrifuged to remove the Triton supernatant, resuspended in a standard activation medium containing unlabelled ATP and protein kinase catalytic subunit instead of cyclic AMP, and incubated for the times ( $\mathrm{min}$ ) shown at the top of the lanes, or for $2 \mathrm{~min}$ in the lanes labelled $-E$ and $-K$. At the end of the incubation period, labelled ATP and $10 \mu \mathrm{l}$ of high-Triton extract (HTE) were added. This mixture was then incubated for an additional $2 \mathrm{~min}$. Control lane $-\mathbf{E}$ shows a sample prepared with $10 \mu \mathrm{l}$ of the $0.1 \%$ Triton solution used to prepare HTE, instead of HTE, and protein kinase catalytic subunit was omitted from the sample in control lane $-K$.

(HTE), prepared as described in Materials and methods, activation of a normal level of motility was observed. Control experiments using an equal volume of the $0.1 \%$ Triton solution used to prepare HTE did not give activation of motility. Addition of cyclic AMP-dependent protein kinase inhibitor, $\mathrm{H} 8$, at a concentration of $0.4 \mathrm{mM}$ just before addition of HTE prevents the activation of motility. The presence of $20 \mu \mathrm{g} \mathrm{ml}^{-1}$ PGT during the second incubation period also prevents the activation of motility. These results suggest that phosphorylations essential for the activation of motility occur during the second incubation period if HTE is present.

We have attempted to identify these phosphorylations by carrying out the first incubation in the presence of unlabelled ATP, and adding $\left[{ }^{32} \mathrm{P}\right] \mathrm{ATP}$ at the start of the second incubation period during which motility is activated. Results are shown in Fig. 6, and in Table 1, line (d). In these experiments, correlation with motility is indicated by higher values in Table 1 . The control lane labelled $-K$, from a sample to which neither cyclic AMP nor protein kinase catalytic subunit were added, demonstrates that the HTE added to the sample does not contain sufficient active kinase to permit a significant level of phosphorylation. The control lane labelled $-E$, from a sample that received $10 \mu l$ of the $0.1 \%$ Triton extraction solution used to prepare HTE, instead of HTE, confirms that almost all of the phosphorylation that could be carried out without HTE was completed after $120 \mathrm{~s}$ of incubation. The 0 min lane yields results that are similar to those obtained under standard conditions, as in lane S of Fig. 1. There is, however, a noticeable decrease in the labelling of bands $\mathrm{N}$ and $\mathrm{O}$. After only $0.5 \mathrm{~min}$ incubation with protein kinase catalytic subunit, there is a major decrease in the subsequent labelling of several bands, indicating that these bands become rapidly phosphorylated in the absence of supernatant, without activation of motility. These bands include the dynein bands $A$ and $R$, as well as bands $\mathrm{D} 1, \mathrm{I}, \mathrm{K}$ and Q. Other experiments with shorter incubation times indicate that the phosphorylation of band $\mathrm{K}$ is particularly rapid, and is essentially complete within $15 \mathrm{~s}$ (not shown). In contrast, some bands such as E, F, J, M2, and, especially, M1 are phosphorylated more slowly during the first incubation with unlabelled ATP, and further phosphorylation occurs during the second incubation period, when motility is activated. This experiment failed to identify any unique phosphoproteins that were phosphorylated only during motility activation during the second incubation period, but did identify band $\mathrm{M} 1$ as a phosphoprotein with a particularly strong correlation with activation of motility.

\section{Discussion}

\section{Phosphorylation of dynein polypeptides during activation} of motility

There has been no previously reported work on isolation or characterization of dynein from Ciona sperm flagella. We have carried out only the minimal characterization necessary to assess the significance of the high molecular mass phosphoprotein (band A). Characterization as dynein is based on extractibility of approximately half of the axonemal ATPase and the most prominent high molecular mass polypeptide band by high salt, as a particle that sediments at about $20 \mathrm{~S}$, along with an array of lower molecular mass components. Under some conditions, the extracted high molecular mass polypeptide band can be resolved into equal $\alpha$ and $\beta$ bands. In a variety of other sperm flagella, these characteristics have been shown to be associated with removal of outer dynein arms from the axoneme and appearance of dynein particles in the supernatant, as judged by electron microscopy (cf. Bell et al. 1982; Witman, 1989). Work in other systems has also shown that both the $\alpha$ and $\beta$ high molecular mass polypeptides are ATPases.

Confirming the suggestion from earlier work by Opresko and Brokaw (1983), we can therefore conclude that one or both of these $\alpha$ and $\beta$ dynein polypeptides of Ciona sperm flagella, probably from the outer dynein arms, become rapidly phosphorylated during both in vitro and in vivo activation of sperm flagellar motility. In Mytilus spermatozoa, the $\alpha$ polypeptide of both inner and outer arm dyneins is reported to be phosphorylated during incubation of axonemes with cyclic AMP and ATP (Stephens and Prior, 1991). The $\alpha$ polypeptide, as well as three smaller subunits ( $72 \mathrm{kDa}, 23 \mathrm{kDa}$ and $18 \mathrm{kDa}$ ), of sea urchin sperm flagellar dynein have been reported to be substrates for phosphorylation when the catalytic subunit of the cyclic AMP-dependent protein kinase is added to isolated dynein (Tash, 1989). This kinase was also able to phosphorylate $22 \mathrm{~S}$ dynein from Tetrahymena cilia to a level of $0.5 \mathrm{~mol}$ of phosphate per mol of dynein, with most 
of the label appearing on intermediate and light chains, and only a small amount on the dynein heavy chains (Chilcote and Johnson, 1990). The $\alpha$ polypeptide, and no other polypeptides, of Chlamydomonas outer arm dynein becomes phosphorylated in vivo, on five or six serine/ threonine residues (King and Witman, 1989). None of these other examples provides the correlation of dynein phosphorylation with activation of motility that is possible with Ciona spermatozoa.

The most rapidly migrating major phosphoprotein of the sperm or axonemal pellets was previously designated as band $\mathrm{R}$, at $18 \mathrm{kDa}$ (Opresko and Brokaw, 1983). In our analyses, a value closer to $20 \mathrm{kDa}$ was indicated. In the earlier work, band $R$, along with the dynein band $A$, was identified as a band that became rapidly phosphorylated during in vivo activation of motility. We have now found that band $R$ is also associated with the $20 \mathrm{~S}$ dynein particle, so that is tentatively identified as a light chain of dynein. However, no band corresponding to phosphoprotein band $R$ is visible in the silver-stained gels, nor do we see in these gels any characteristic pattern of dynein light chains similar to those seen in other work (Bell et al. 1982; Gatti et al. 1989). The strong phosphorylation of this dynein light chain suggests that it might have a regulatory function, based on the analogy with regulatory function that has been indicated for myosin light chain phosphorylation. Phosphorylation of dynein intermediate and light chains has been reported in several other flagellar and ciliary systems (Bonini and Nelson, 1990; Hamasaki et al. 1989; Hamasaki and Satir, 1989; Stephens and Prior, 1990; Stommel and Stephens, 1985), but in this previous work it was not possible to demonstrate a correlation with activation of motility.

On the other hand, the idea that these phosphorylations of dynein components, bands $\mathrm{A}$ and $\mathrm{R}$, are associated with activation of motility is not sustained by experiments of the type illustrated in Fig. 6. These experiments demonstrate that when spermatozoa that have been centrifuged to remove the Triton supernatant are incubated with catalytic subunit of cyclic AMP-dependent protein kinase in the absence of supernatant, bands $A$ and $R$ become thoroughly phosphorylated in $2-5 \mathrm{~min}$, but without any activation of flagellar motility. This coincides with previous work showing that these bands become labelled, without activation of motility, when washed spermatozoa are incubated with the kinase in the presence of labelled ATP (Opresko and Brokaw, 1983). The new result is more incisive, because it argues that the absence of motility in these experiments is not the result of only partial phosphorylation or phosphorylation of polypeptides A and $R$ on the 'wrong' sites, in the absence of supernatant. The conclusion must be that, although phosphorylation of these two dynein polypeptides occurs during activation of motility, it is not sufficient for activation of motility of these flagella. We cannot say whether phosphorylation of these dynein polypeptides is necessary for activation of motility.

The most obvious mechanism for activation of sperm flagellar motility would be conversion of dynein from an inactive state to an active state that was capable of generating the sliding that is the basis for flagellar bending. The simplest form of such a model would suggest that every dynein would need to be in a dephosphorylated state when motility was inactivated, and would need to be phosphorylated upon activation. This would predict a much higher level of ${ }^{32} \mathrm{P}$ incorporation than we observed for the dynein heavy chain. However, if this dynein heavy chain is constitutively phosphorylated, it is possible that a small number of dephosphorylated dyneins could cause the formation of stable cross-bridges that prevent sliding, and activation might be accomplished by a small amount of dynein heavy-chain phosphorylation.

There are other indications that activation of flagellar motility is not mediated by activation of sliding. Inactive Ciona spermatozoa, demembranated and exposed briefly to trypsin, disintegrate by active sliding when ATP is added (Brokaw, 1982). This could be the result of removal of a regulatory element by trypsin, but demembranated, inactive Ciona sperm show very little activation of motility when exposed to trypsin in the presence of ATP (Brokaw, 1982), in contrast to results with trout spermatozoa (Okuno and Morisawa, 1982) or quiescent sea urchin spermatozoa (Brokaw, 1985; Gibbons and Gibbons, 1984). Detailed studies of the effects of cyclic AMP incubation and protein phosphatase treatment on the movement parameters of sea urchin and Ciona spermatozoa (Brokaw, $1987 b$ ) suggested that phosphorylation was not regulating sliding velocity, but instead was activating a regulatory system controlling flagellar oscillation. Our phosphorylation results suggest that if any axonemal regulatory protein is phosphorylated at the level of one mole of phosphate added per mole of protein during activation of motility, the amount of this regulatory protein in the axoneme must be significantly less than one molecule per dynein arm

\section{Other phosphorylations are required for activation of motility}

Our experiments with spermatozoa centrifuged to removed the Triton supernatant also showed that activation of motility during the second incubation, after addition of a concentrated Triton extract (HTE) of whole spermatozoa, is still inhibited when the kinase inhibitor, $\mathrm{H} 8$, is present during the second incubation. Additional phosphorylation, dependent upon the presence of a supernatant component or components, appears to be required for activation of motility. However, the labelling that is seen during the second incubation period simply completes the phosphorylation of bands such as M1 that become partially labelled in the absence of HTE. The experiment was designed to identify phosphoprotein bands that would appear only when HTE was added, and motility was activated, but this result was not obtained.

Phosphorylation(s) important for activation of motility that occur during the second incubation, in the presence of HTE, might be phosphorylations of supernatant components that are not found in the sperm pellet fractions analysed in Fig. 6. Autoradiograms of gels of the supernatant obtained after the second incubation (not shown here) revealed a large array of phosphoproteins, most or all of which should represent phosphorylation of proteins added with the HTE, but this experiment did not provide any basis for identifying any particular supernatant phosphoproteins that might be important for activation of motility. Attempts to 'activate' HTE by incubation with cyclic AMP or kinase, followed by addition to spermatozoa in the presence of protein kinase inhibitor to inhibit further phosphorylation, have not been successful (Opresko and Brokaw, 1983, and additional unpublished experiments) with Ciona spermatozoa, although successful results from experiments of this type have been reported in other species (Ishiguro et al. 1982; Tash et al. 1986). 
A possible role for tyrosine kinase

We have used an inhibitor, PGT, that has been shown to inhibit the tyrosine phosphorylation of a $15 \mathrm{kDa}$ phosphoprotein of trout spermatozoa that has been associated with activation of sperm motility (Hayashi et al. 1987). This inhibitor has also been shown to inhibit tyrosine phosphorylation in other systems (Braun et al. 1984). Because its inhibition depends upon its content of tyrosine, it is not expected to inhibit serine/threonine kinases, and we have confirmed under the conditions of our experiments that it does not inhibit the phosphorylation of a variety of substrates by the catalytic subunit of the cyclic AMPdependent protein kinase (Fig. 4). This provides strong reason to believe that inhibition by PGT of phosphorylation and activation of sperm motility is the result of inhibition of tyrosine kinase activity, and that phosphorylation of tyrosine residues may be an essential step in the activation of Ciona sperm motility. However, the possibility that PGT is inhibiting a multispecific kinase, such as the kinase described by Featherstone and Russell (1991), that can phosphorylate tyrosine as well as carrying out serine/threonine phosphorylations that are required for activation of motility has not been excluded.

If all of the phosphorylation inhibited by PGT (Fig. 1) were phosphorylation of tyrosine residues, we would have expected that our immunoblotting analyses using antiphosphotyrosine antibodies would have revealed strong effects of PGT on bands identified as phosphotyrosine containing proteins. This was not found.

Our results could be explained by a kinase cascade, with all of the PGT-sensitive flagellar phosphoproteins resulting from activity of endogenous serine/threonine kinase(s), activated by a small amount of tyrosine phosphorylation, which is in turn activated by phosphorylation by either endogenous or exogenous cyclic AMPdependent kinase. We have little evidence that any of the phosphoprotein bands that we describe are the direct result of phosphorylation by the cyclic AMP-dependent kinase, although in vitro phosphorylation of partially purified dyneins by cyclic AMP-dependent kinase has been demonstrated (Chilcote and Johnson, 1990; Tash, 1989). Our quantitation did not identify any major phosphoprotein bands that were labelled equally strongly in the presence of PGT or PGA. Some of the phosphorylations that are strongly inhibited by PGT, such as bands A and R, are bands that are rapidly phosphorylated when washed, demembranated spermatozoa are incubated with cyclic AMP-dependent kinase in the absence of supernatant. Since the phosphorylation of these bands in the absence of supernatant is also inhibited by PGT, the tyrosine kinase and other downstream kinases would appear to be tightly bound to the demembranated spermatozoa. Yokota and Mabuchi (1990) reported association of a major part of the cyclic AMP-dependent protein kinase of sea urchin sperm flagella with the flagellar axonemes. Chilcote and Johnson (1990) reported that endogenous kinase activity was present in their sucrose gradient-purified dynein fractions from Tetrahymena cilia.

The finding that PGT inhibits the activation of motility that occurs after addition of HTE to washed spermatozoa that have been incubated with cyclic AMP-dependent protein kinase, as well as inhibiting phosphorylations that occur before addition of HTE, suggests that the continued presence of a tyrosine kinase activity may be required, to maintain an appropriate level of tyrosine phosphorylation in the presence of tyrosine phosphatase activity.

PGT has also been reported to inhibit tyrosine protein phosphatase activity (Tonks et al. 1988), providing an alternative possibility for a kinase cascade. However, this inhibition of tyrosine protein phosphatase appears to be a general polyanionic effect that would be inconsistent with the strong discrimination between the effects of PGT and PGA that is seen in our experiments. Although none of our data otherwise discriminate between PGT inhibition of a kinase or a phosphatase, the work of Hayashi et al. (1987) on activation of trout sperm motility clearly demonstrated tyrosine phosphorylation of the $15 \mathrm{kDa}$ polypeptide in response to cyclic AMP, and inhibition of this phosphorylation by PGT.

\section{Conclusion: another candidate for a regulatory phosphoprotein}

These experiments identify a $53 \mathrm{kDa}$ phosphoprotein found in demembranated sperm pellet fractions, band M1, as a particularly strong candidate for a phosphoprotein that must be phosphorylated in order to activate motility. This band is least thoroughly phosphorylated by cyclic AMP-dependent kinase in the absence of supernatant (Fig. 6) and accepts further phosphate residues during subsequent activation of motility in the presence of supernatant. Band M1 is strongly phosphorylated during in vivo activation, perhaps more strongly than bands $A$ and R (Fig. 5). Phosphorylation of band M1 is strongly inhibited by PGT (Fig. 1). Several other bands, such as band F, share these characteristics, but to a somewhat lesser degree.

Although bands M1 and M2 were not separately identified by Opresko and Brokaw (1983), inspection of Figure 7 of that paper indicates that it is the upper part of band $\mathrm{M}$, corresponding to our band M1, that is found after labelling of isolated demembranated flagella, so band M1 is likely to be an axonemal phosphoprotein. Phosphoprotein band M1 cannot be clearly correlated with a band in the silver-stained gels.

We thank Holly Dodson and Jim Pacheco for collecting Ciona and other assistance with these experiments. This work has been support by a grant from the National Institutes of Health, GM18711 .

\section{References}

Bell, C. W., Fraser, C. L., Sale, W. S., Tang, W-J. Y. and Gibbons, I. R. (1982). Preparation and purification of dynein. Meth. Enzym. 85, $450-474$

Bonini, N. M. and Nelson, D. L. (1990). Phosphoproteins associated with cyclic nucleotide stimulation of ciliary motility in Paramecium. J. Cell Sci. 95, 219-230.

Braun, S., RaYmond, W. E. AND Racker, E. (1984). Synthetic tyrosine polymers as substrates and inhibitors of tyrosine-specific protein kinases. J. biol Chem 259, 2051-2054.

Brokaw, C. J. (1982). Activation and reactivation of Ciona spermatozoa. Cell Motil. Suppl. 1, 185-189.

Brokaw, C. J. (1985). Cyclic AMP-dependent activation of sea urchin and tunicate sperm motility. Ann. N. Y. Acad. Sci. 438, 132-141.

Brokaw, C. J. (1987a). Regulation of sperm flagellar motility by calcium and cAMP-dependent phosphorylation. J. cell. Biochem. $\mathbf{3 5}$, 175-184

BroKAw, C. J. (1987b). A lithium-sensitive regulator of sperm flagellar oscillation is activated by cAMP-dependent phosphorylation. J. Cell Biol. 105, 1789-1798.

Brokaw, C. J. (1992). Activation of motility of Clona spermatozoa. In Comparative Spermatology 20 Years After (ed. B. Baccetti), Rome, Serono Symposia (in press).

Carter, S. G. AND KarL, D. W. (1982). Inorganic phosphate assay with malachite green: an improvement and evaluation. $J$. blophys. biochem. Meth. 7, 7-13.

Chilcote, T. J. AND Johnson, K. A (1990). Phosphorylation of Tetrahymena $22 \mathrm{~S}$ dynein. J. biol. Chem. 265, 17 257-17266. 
de la Houssaye, B. A. and Masaracchia, R. A. (1983). Standardization of the assay for the catalytic subunit of cyclic AMP-dependent protein kinase using a synthetic peptide substrate. Analyt. Biochem. 128 54-59.

DEY, C. S. AND BRoKaw, C. J. (1989). Tyrosine phosphorylation is required for activtion of Ciona sperm motility. J. Cell Biol. 109, 178A.

Featherstone, C. and Russell, P. (1991). Fission yeast $\mathrm{p} 107^{\mathrm{wee} 1}$ mitotic inhibitor is a tyrosine/serine kinase. Nature 349, 808-811.

Gatti, J-L., King, S. M., Moss, A. G. and Witman, G. B. (1989). Outer arm dynein from trout spermatozoa. J. bool. Chem. 264, 11450-11 457.

Gibbons, B. H. ANd Gibbons, I. R. (1984). Lithium reversibly inhibits microtubule-based motility in sperm flagella. Nature 309, 560-562.

Hamasaki, T., Murtaugh, T. J., SatiR, B. H. and Satir, P. (1989). In vitro phosphorylation of Paramecum axonemes and permeabilized cells. Cell Motil. Cytoskel. 12,1-11.

Hamasaki, T. and Satir, P. (1989). Parameclum dynein contains a phsophorylatable $29 \mathrm{KDa}$ light chain. Cell Motil. Cytoskel. 11, $189 \mathrm{~A}$

Hayashi, H., Yamamoto, K., Yonekawa, H. and Morisawa, M. (1987) Involvement of tyrosine protein kinase in the initiation of flagellar movement in rainbow trout spermatozoa. J. bıol. Chem. 262, 16692-16698.

Hidaka, H., Inagakt, M., Kawamoto, S. ANd Sasaki, Y. (1984). Isoquinolinesulfonamides, novel and potent inhibitors of cyclic nucleotide dependent protein kinase and protein kinase $C$. Biochemistry 23, 5036-5041.

Ishiguro, K., Murofushi, H. ANd SAKaI, H. (1982). Evidence that cAMP-dependent protein kinase and a protein factor are involved in reactivation of Triton X-100 models of sea urchin and starfish spermatozoa. J. Cell Brol. 92, 777-782.

KING, S. M., OTtER, T. and Witman, G. E. (1985). Characterization of monoclonal antibodies against Chlamydomonas flagellar dyneins by high-resolution protein blotting. Proc. natn. Acad. Sci. U.S.A. 82 4717-4721.

King, S. M. and Witman, G. B. (1989). Molecular structure of Chlamydomonas outer arm dynein. In Cell Movement, vol. 1, The Dynein ATPases (ed. F. D. Warner, P. Satir, and I. R. Gibbons), pp. 61-75. New York: A. R. Liss, Inc.

Kitazoe, Y., Miyahara, M., Hiraoka, N., Ueta, H. and Utsumi, K. (1983). Quantitative determination of overlapped proteins in sodium dodecyl sulfate-polyacrylamide gel electrophoresis. Analyt. Biochem. 134, 295-302.

LAEMMLI, U. K. (1970). Cleavage of structural proteins during assembly of bacteriophage T4. Nature 227, 680-685.

Morisawa, M. and Hayashi, H. (1985). Phosphorylation of a $15 \mathrm{~K}$ axonemal protein is the trigger initiating trout sperm motility. Biomed. Res. 6, 181-184.

Morisawa, M. AND OKuno, M. (1982). Cyclic AMP induces maturation of trout sperm axoneme to initiate motılity. Nature 295, 703-704.

MORRISSEY, J. H (1981). Silver stain for proteins in polyacrylamide gels: a modified procedure with enhanced uniform sensitivity. Analyt. Blochem. 117, 307-310.

OKuno, M. And Morisawa, M. (1982). Cyclic AMP and initiation of flagellar movement in rainbow trout spermatozoa. In Brological Functions of Microtubules and Related Structures (ed. H. Sakai, G. G. Borisy, and H. Mohri), pp. 151-163. Tokyo and New York: Academic Press.

Opresko, L. AND Brokaw, C. J. (1983). cAMP-dependent phosphorylation associated with activation of motility of Ciona sperm flagella. Gamete Res. 8, 201-218.

Peaucellier, G., Veno, P. A. And Kinsey, W. H. (1988). Protein tyrosine phosphorylation in response to fertilization. J. bool. Chem 263, $13806-13811$

Stephens, R. E. ANd Prior, G. (1990). Cyclic AMP-dependent dynein light chain phosphorylation in neuronally-controlled mussel gill cilia. J. Cell Biol. 111, 295A.

Stephens, R. E. AND Prior, G. (1991). Cyclic AMP-dependent phosphorylation of dynein alpha-heavy chains in Mytllus edulis sperm flagella. Biophys. J. 59, 567A

Stommel, E. W. ANd Stephens, R. E. (1985). Cyclic AMP and calcium in the differential control of Mytilus gill cilia. J. comp. Physiol. A 157, 451-459.

TASH, J. S. (1989). Protein phosphorylation: the second messenger signal transducer of flagellar motility. Cell Motil. Cytoskel. 14, 332-339.

Tash, J. S., Hidaka, H. AND Mrans, A. R. (1986). Axokinin phosphorylation by cAMP-dependent protein kinase is sufficient for activation of sperm flagellar motility. J. Cell Bol. 103, 649-655.

Tonks, N. K., Diltz, C. D. AND Fischer, E. H. (1988). Characterization of the major protein-tyrosine phosphatases of human placenta. J. biol Chem. 263, 6731-6737.

Witman, G. B. (1989). Perspective: Composition and molecular organization of the dyneins. In Cell Movement, vol. 1, The Dynein ATPases (ed. F. D. Warner, P. Satir, and I. R. Gibbons), pp. 25-35. New York: A. R. Liss, Inc.

Yokota, E. AND MABUCHI, I. (1990). The cAMP-dependent protein kinase in sea urchin sperm tails: association of the enzyme with the flagellar axonemes. J. Biochem. 108, 1-3.

(Received 16 July 1991 - Accepted 20 August 1991) 